\title{
Review of: "Tarsal morphology of ischyromyid rodents from the middle Eocene of China gives an insight into the group's diversity in Central Asia"
}

\author{
Laura Stroik ${ }^{1}$ \\ 1 Grand Valley State University
}

Potential competing interests: The author(s) declared that no potential competing interests exist.

This study addresses an important gap in our knowledge of rodent evolution: the postcranium of early Asian rodents (ischyromyids). Although the postcranial material presented here is limited, it provides an interesting first glimpse into the locomotor and substrate use components of the early Asian rodent ecological niche. Furthermore, the discussion of the presence of three distinct taxa allows for preliminary interpretation of Asian ischyromyid community structure at this time.

Overall, the manuscript is clear, concise, and well-written, but I include a few areas for (minor) revision below:

The use of "paleoecology" in the last sentence of the first paragraph of p. 2 is a bit strange. Perhaps "ecomorphology" would be more appropriate? In either case, I think this needs to be restated and explained a bit further.

The "Systematic remark" for IVPP V24417 is a bit confusing. The attribution of the specimen to Asiomys dawsoni is understandable based on its in situ association with dental material. However, the argument that its "distinctive size" was a contributing factor in this attribution is not clear, particularly as Species A and Species B are also large specimens (and even larger than IVPP V24417).

Why was IVPP V24417 not included in the PCA? Is this the result of its more fragmentary nature? If so, would it be possible to perform a PCA on the entire sample in addition to this specimen (and only include those measurements that can be taken on all specimens)? It would be interesting to see how all three fossil specimens fall out in the PCA results (unless there is a reason this wasn't done originally).

In the discussion of the PCA results, it is stated: "The strong negative correlation of the length of the calcaneal body in the second component is illustrated by grouping the species with a strong jumping locomotor repertoire (e.g., squirrels and chinchillas) towards the left side of the plot." However, the left side of the plot should refer to negative values of PC1. Please reinterpret these results given this 
information.

I think the Discussion could benefit from a further (or more explicit) explanation of:

1. The connections among calcaneal morphology, locomotor niche, and community structure of this Asian ischyromyid assemblage and those of $\mathrm{N}$. America and Europe. This information is present in pieces, but stronger links among these components and a clearer comparison with other communities would be helpful. I realize that the fossil material is limited but even a first evaluation of this would be interesting here (with caveats).

2. Ischyromyid competitors. You mention potential competitors of the Asian ischyromyids, but this is based mainly on body size (first molar length). How do your locomotor reconstructions affect your assessment of competition among these taxa?

For clarity, please include the orientation information (e.g., dorsal, medial, etc.) for the images in Fig. 2 and the line drawings of Fig. 3 (as was done for the photos of Fig. 3).

I think replacing the variable numbers with the abbreviations used in Table 1 would help with ease of interpretation of Fig. 4. At a minimum, there needs to be a key that relates the variable numbers to the names. 